\title{
Gravitation Theory Based on the Physics of an Absolute Reference System
}

\author{
Konstantinos Patrinos \\ National Technical University of Athens, Athens, Greece \\ Email:kpatr@central.ntua.gr
}

How to cite this paper: Patrinos, K. (2021) Gravitation Theory Based on the Physics of an Absolute Reference System. Journal of Applied Mathematics and Physics, 9, 11941214.

https://doi.org/10.4236/jamp.2021.96082

Received: May 3, 2021

Accepted: June 8, 2021

Published: June 11, 2021

Copyright (c) 2021 by author(s) and Scientific Research Publishing Inc. This work is licensed under the Creative Commons Attribution International License (CC BY 4.0).

http://creativecommons.org/licenses/by/4.0/

\section{(c) (i) Open Access}

\begin{abstract}
The study of the effect of the gravitational field on the photons, based on the hypothesis of the absolute reference system, demonstrates the origin of the gravitational force. By studying the propagation of a photon in the gravitational field the change in the estimation of time is determined, resulting from the use of a clock that is affected by the gravitational field. It is proved that in all known experiments, which were carried out in order to confirm the general theory of relativity, the results based on the hypothesis of an absolute reference system agree with the corresponding results of the general theory of relativity, except for the result of the deflection of light in the gravitational field of the sun, where the experimental results confirm the hypothesis of the absolute reference system.
\end{abstract}

\section{Keywords}

Gravitational Field, Gravitational Time Dilation, Gravitational Redshift, Perihelion of Mercury, Gravitational Deflection of Light, Time Delay of Light, Gravitational Redshift

\section{Introduction}

The motivation for the study presented in this article is to examine the agreement of the hypothesis of the absolute reference system with the already obtained experimental results that have emerged from the relevant tests of the general theory of relativity. In order to carry out this examination, we first study the effect of the gravitational field on a photon, and then we study the corresponding effect of the gravitational field on material bodies, since, based on the hypothesis of the absolute reference system, the elementary particles of matter are composed of bound photons. This study contributes to the formulation of a gravitational theory that fully meets the requirements expressed by all the concerns 
raised in matters related to the examined effects of the gravitational field.

Using the principles of the hypothesis of an absolute reference system, we first consider the origin of the attracting force of a gravitational field coming from a spherical homogeneous body of mass $M$. By this study emerge the characteristics of the effect of the gravitational field on the photons that propagate in the space around this body. A photon, which is affected by this gravitational field, behaves as a particle with mass $m=h v / c^{2}$, that is, an attracting gravitational force is exerted on it, which is analogous to that acting on all bodies, and in addition, the velocity, the mass, and also all physical quantities involved in determining the state of the photon are functions of the radial distance of the photon from the center of the spherical body.

Due to the microstructure of the elementary particles of matter, i.e. the bound photons that are the building elements of the particles, according to the hypothesis of the absolute reference system, the aforementioned changes in the state of a photon cause the change in the estimation of time, resulting from the use of a clock which is affected by the gravitational field. Based on this change in the estimation of time, all the values of the physical quantities that characterize the photonic state are redefined.

Using all these theoretical results, which are taken by the formulation of the physics of an absolute reference system in [1] [2] [3], we study in the last section the experimental tests of the general theory of relativity in order to test experimentally the hypothesis of an absolute reference system.

The history of the tests of the general theory of relativity begins with the three corresponding propositions of Albert Einstein in 1915, and these tests concerned the precession of the perihelion of Mercury, the deflection of light in gravitational fields, and the gravitational redshift. The advance of the perihelion of Mercury was already known in the nineteenth century. Experiments showing light bending were performed in 1919. Measurements of the gravitational redshift have taken in 1925, although measurements that actually confirm the theory were not made until 1954. A more accurate program starting in 1959 tested general relativity in the weak gravitational field limit, severely limiting possible deviations from the theory. In the 1970s, was made additional tests, with measurement of the relativistic time delay in radar signal travel time near the sun, by Irwin Shapiro. In 1974, Hulse, Taylor and others studied the behaviour of binary pulsars. Both in the weak field limit and with the stronger fields present in systems of binary pulsars the predictions of general relativity have been extremely well tested.

\section{The Origin of Attractive Force in a Gravitational Field}

Considering that the elementary electromagnetic oscillation of a photon is the oscillation of the ether, as a means of propagating electromagnetic wave, it follows that the radial propagation of photons, which are force carriers of the gravitational field of a spherical body of mass $M$, makes the ether surrounding the 
body a medium which is in a constant state of oscillation.

Therefore, if a photon comes from a space away from the body of mass $M$, so that it can practically be thought to come from a space outside the gravitational field, and propagates in the gravitational field, then this photon will be affected by the pre-existing oscillation of the propagation medium, i.e. will be affected by the oscillation of the ether.

In the next two sections the clock which is used to measure speed, momentum, force and energy inside and outside the gravitational field is currently a clock which is outside the gravitational field. We also consider that outside the gravitational field is a body or a measuring instrument when it is very far from the body to which the gravitational field is due.

Based on the hypothesis of the absolute reference system, the elementary photonic wave is considered to be an oscillator whose average value of kinetic energy is equal to $(1 / 2) h v$, where $h$ is Plank's constant and $v$ is the frequency, and is also equal to the mean value of the dynamic energy ([2], Subsection 3.3. Harmonic Oscillator). The total energy of a photon will be equal to $h v$. Also according to the section "Introduction to Particle Mechanics" in [1], we have the relation $h v=m_{p h} c^{2}$, where $m_{p h}$ is the photonic mass and $c$ is the speed of light in vacuum. We must then consider the effect of the gravitational field on a photon coming from a space in which there is no gravitational field, and then propagates in a gravitational field, that is, on the already disturbed ether.

Based on the intrinsic properties of the ether, a portion of the oscillation of the aforementioned photon, equivalent to an amount of energy, $E_{p h}$, is subtracted from the original photon energy it had when it was outside the gravitational field. This amount of energy, $E_{p h}$, is added locally to the gravitational field energy. Under these conditions the photonic energy decreases in the gravitational field and therefore the photonic mass and the photonic speed will be functions of the radial distance as we will see below. Due to this change we will then denote by $m_{p h_{o}}, c_{o}$ and $v_{o}$ the mass, velocity and frequency of the photon outside the gravitational field, while inside the gravitational field these quantities will be denoted by $m_{p h}, c$ and $v$ respectively for the same photon. Since the sum of the total photon energy, $m_{p h} c^{2}$, inside the gravitational field and the energy $E_{p h}$ gives the total photon energy that the photon had outside the gravitational field, the relation of the photonic energies outside and inside the gravitational field is:

$$
m_{p h_{o}} c_{o}^{2}=E_{p h}+m_{p h} c^{2}
$$

We will then examine the force exerted on the aforementioned photon in the gravitational field due to the existence of a spherical homogeneous celestial body. The mass of the spherical body is denoted by $M$. The energy $E_{p h}$ depends on the local density of the oscillating force carriers of the gravitational field, which are photons as we mentioned before, so it depends on the radial distance $r$ from the center of the spherical body. Therefore the quantities $m_{p h}, c$ and $v$ will be 
functions of the radial distance $r$. The dependence of the energy $E_{p h}$ on the radial distance $r$ is the cause of the existence of the gravitational force, which is exerted on the aforementioned photon. The work of this force along a vector differential displacement $\mathrm{d} \boldsymbol{r}$ is $\boldsymbol{F} \cdot \mathrm{d} \boldsymbol{r}=\mathrm{d} E_{p h}$. This means that at a given radial distance there will be a gradient of the photonic energy, which causes a force dependent on this position. In polar coordinates $r, \theta$, with unit vectors $\hat{u}_{r}, \hat{u}_{\theta}$, the vector differential displacement is $\mathrm{d} \boldsymbol{r}=\mathrm{d} r \hat{u}_{r}+r \mathrm{~d} \theta \hat{u}_{\theta}$, so for $\boldsymbol{F}=f(r) \hat{u}_{r}$ gives us $\boldsymbol{F} \cdot \mathrm{d} \boldsymbol{r}=f(r) \mathrm{d} r$. Therefore the function of the force $f(r)$, which is the gradient of the photonic energy we talked about before, will be given by the relation:

$$
f(r)=\frac{\mathrm{d} E_{p h}}{\mathrm{~d} r}
$$

If at radial distance $r$ the energy $E_{p h}$ is expressed as $E_{p h}(r)$, while at radial distance $r+\mathrm{d} r$ is expressed as $E_{p h}(r+\mathrm{d} r)$, then for $\mathrm{d} r>0$, based on what we said before for the dependence of the energy $E_{p h}$ on the distance $r$, it follows that $E_{p h}(r+\mathrm{d} r)<E_{p h}(r)$, i.e. $\mathrm{d} E_{p h}<0$, while for $\mathrm{d} r<0$ it follows that $\mathrm{d} E_{p h}>0$. Therefore the values of the function of the force, $f(r)$, are negative.

Also the relation (1) give us the differential equation $\mathrm{d} E_{p h}+\mathrm{d}\left(m_{p h} c^{2}\right)=0$, and therefore, according to the expression (2) of the gradient of the photonic energy, we take the equation:

$$
f(r) \mathrm{d} r+\mathrm{d}\left(m_{p h} c^{2}\right)=0
$$

As already shown in [1] (Subsection 2.4. Electromagnetic Field and Photons, Equation (2.33)) the density of the force carriers emitted at a constant rate, in this case from the spherical body, is inversely proportional to the square of the radial distance, and is also proportional to the force exerted by the gravitational field. Therefore the function $f(r)$, which expresses the gravitational force due to the gravitational field of a spherical homogeneous body, will be proportional to the quantity $1 / r^{2}$ and will also be proportional to the masses $M$ and $m_{p h}$. So, since the values of $f(r)$ are negative, the force vector will obey the relation:

$$
\boldsymbol{F}=f(r) \hat{u}_{r}=-G \frac{M m_{p h}}{r^{2}} \hat{u}_{r}
$$

which is the well-known Newtonian gravitational force, and $G$ is the known universal gravitational constant. According to the hypothesis of the absolute reference system, all the elementary particles of matter are composed of bound photons ([1], Subsection 2.4.1. Contraction of Length and Time). Therefore, in the gravitational field, this force is exerted on all bound photons which are the building elements of the body. It follows that if the distance between the centers of two spherical homogeneous bodies, with masses $M$ and $m$, is equal to $r$, the gravitational force is given by the known relation:

$$
\boldsymbol{F}=-G \frac{M m}{r^{2}} \hat{u}_{r}
$$




\section{Mass and Velocity of a Photon in the Gravitational Field}

The momentum of the photon, without the presence of a gravitational field, making use of the clock which is outside the gravitational field, is given by the relation $p_{p h_{o}}=m_{p h_{o}} c_{o}$ ([1] section 4. Introduction to Particle Mechanics). The corresponding relation for a photon propagating in a gravitational field and resulting from the use of the aforementioned clock, in vector form, is:

$$
\boldsymbol{p}_{p h}=m_{p h} \boldsymbol{c}
$$

where $m_{p h}, \boldsymbol{c}$ are functions dependent on the radius $r$. We consider that the photon propagates in space with the simultaneous presence of a gravitational field derived from the spherical body of mass $M$. Therefore, for a vector differential displacement $\mathrm{d} \boldsymbol{r}$, given that $\boldsymbol{c}=\mathrm{d} \boldsymbol{r} / \mathrm{d} t$, the differential $\boldsymbol{F} \cdot \mathrm{d} \boldsymbol{r}$ is calculated as follows:

$$
\begin{aligned}
\boldsymbol{F} \cdot \mathrm{d} \boldsymbol{r} & =\frac{\mathrm{d} \boldsymbol{p}_{p h}}{\mathrm{~d} t} \cdot \mathrm{d} \boldsymbol{r} \\
& =\frac{\mathrm{d}\left(m_{p h} \boldsymbol{c}\right)}{\mathrm{d} t} \cdot \mathrm{d} \boldsymbol{r} \\
& =\mathrm{d} m_{p h} \boldsymbol{c} \cdot \boldsymbol{c}+m_{p h} \boldsymbol{c} \cdot \mathrm{d} \boldsymbol{c} \\
& =\mathrm{d} m_{p h} c^{2}+m_{p h} c \mathrm{~d} c
\end{aligned}
$$

According to the relations (4) and (7), given that $\mathrm{d} r=\mathrm{d} r \hat{u}_{r}+r \mathrm{~d} \theta \hat{u}_{\theta}$, we get the relation:

$$
-G \frac{M}{r^{2}} \mathrm{~d} r=\frac{\mathrm{d} m_{p h}}{m_{p h}} c^{2}+c \mathrm{~d} c
$$

According to the relation (4) the function of the gravitational force exerted on the aforementioned photon is given by the relation $f(r)=-G M m_{p h} / r^{2}$. Therefore, using the relation (3), we get the following equality:

$$
G \frac{M}{r^{2}} \mathrm{~d} r=\frac{\mathrm{d}\left(m_{p h} c^{2}\right)}{m_{p h}}=\frac{\mathrm{d} m_{p h}}{m_{p h}} c^{2}+2 c \mathrm{~d} c
$$

After the subtraction of Equations (8) and (9), the integration for radial distance from $r$ to $\infty$ give us:

$$
\int_{c^{2}}^{c_{o}^{2}} \mathrm{~d} v^{2}=4 G m_{p h} \int_{r}^{\infty} \frac{\mathrm{d} x}{x^{2}} \Leftrightarrow c=c_{o}\left(1-\frac{4 G M}{r c_{o}^{2}}\right)^{1 / 2}
$$

which means that the velocity of the photon decreases as it enters the gravitational field. By doubling the two members of the relation (8) and subtracting them from the two members of the relation (9) we get the equality:

$$
\frac{\mathrm{d} m_{p h}}{m_{p h}}=-3 \frac{G M}{c^{2} r^{2}} \mathrm{~d} r
$$

so substituting the quantity $c^{2}$, according to the relation (10), the integration give us:

$$
\int_{m_{p h}}^{m_{p h_{o}}} \frac{\mathrm{d} m}{m}=\int_{r}^{\infty} \frac{-3 G M \mathrm{~d} x}{x\left(x c_{o}^{2}-4 G M\right)} \Leftrightarrow m_{p h}=m_{p h_{o}}\left(1-\frac{4 G M}{r c_{o}^{2}}\right)^{-3 / 4}
$$


that is, in contrast to what happens with the speed of a photon, its mass increases as it enters the gravitational field. Also since the elementary particles have as structural elements the bound photons, the function of the photonic mass, which is due to the dependency on the radial distance, will be analogous to the corresponding function of the mass of a body located inside the gravitational field. If $m$ and $m_{o}$ are the masses of a spherical body inside and outside the gravitational field respectively, and $r$ is the radial distance, the mass function $m$ is given by the relation:

$$
m=m_{o}\left(1-\frac{4 G M}{r c_{o}^{2}}\right)^{-3 / 4}
$$

The dependence of mass on radial distance changes the equation of motion of a body, or a photon, that moves under the influence of the gravitational field. As it turns out easily, the orbital angular momentum remains constant during the movement, since the gravitational force is central. The equation of motion, for a velocity $v$ much less than the speed of light in vacuum, is given by the relation:

$$
\boldsymbol{F}=\dot{m} \boldsymbol{v}+m \dot{\boldsymbol{v}}
$$

where $\dot{m}=\mathrm{d} m / \mathrm{d} t$ and $\dot{\boldsymbol{v}}=\mathrm{d} \boldsymbol{v} / \mathrm{d} t$. Since in polar coordinates the velocity and acceleration are given by the relations $v=\dot{r} \hat{u}_{r}+r \dot{\theta} \hat{u}_{\theta}$ and $\dot{\boldsymbol{v}}=\left(\ddot{r}-r \dot{\theta}^{2}\right) \hat{u}_{r}+(2 \dot{r} \dot{\theta}+r \ddot{\theta}) \hat{u}_{\theta}$ respectively, the equation of motion under the influence of the central gravitational force, according to Equation (5), is given by the relation:

$$
-G \frac{M m}{r^{2}}=\dot{m} \dot{r}+m \ddot{r}-m r \dot{\theta}^{2}
$$

In order to determine the differential equation of the trajectory and the corresponding solution, which is the radial distance as a function of the polar angle $\theta$ we will replace the operator $\frac{\mathrm{d}}{\mathrm{d} t}$ with its equivalent $\frac{L}{m r^{2}} \frac{\mathrm{d}}{\mathrm{d} \theta}$, where $L$ is the constant angular momentum ([4], 3-5 The Differential Equation for the Orbit, and Integrable Power-Law Potentials, Equation (3-32)) and in addition we will change the variable $r$ with the variable $u$ according to the relation $u=1 / r$. Making these substitutions, the differential Equation (14) becomes:

$$
\frac{\mathrm{d}^{2} u}{\mathrm{~d} \theta^{2}}+u=\frac{G M m^{2}}{L^{2}}
$$

Equation (15) is similar to the corresponding equation of classical mechanics ([4], 3-7 The Kepler Problem: Inverse Square Law of Force, Equation (3-50)), but there is a substantial difference. The mass $m$ of Equation (15) is a function of radial distance, while the mass of classical mechanics is constant.

For a photon propagating in the gravitational field, according to the hypothesis of the absolute reference system, the procedure for determining the orbital equation will be the same as the previous one. Therefore, it will obey the relation: 


$$
\frac{\mathrm{d}^{2} u}{\mathrm{~d} \theta^{2}}+u=\frac{G M m_{p h}^{2}}{L^{2}}
$$

\section{Gravitational Time Dilation and Redshift}

In the previous two sections, all values of velocity, momentum, force and energy, for phenomena occurring within the gravitational field, are obtained using a clock outside the gravitational field. What we will examine in this section is the estimation of time with a clock inside the gravitational field, and the effect that this redefinition of estimated time has on the estimation of all the aforementioned physical quantities.

For the time being, however, let us continue to estimate time using the clock that is outside the gravitational field. We have already calculated the changes in mass and velocity of a photon resulting from its entry into a gravitational field. The corresponding change in frequency is determined by the relation $m_{p h} c^{2}=h v$. From the relations (10) and (11) the following relation emerges:

$$
m_{p h} c^{2}=h v=m_{p h_{o}} c_{o}^{2}\left(1-\frac{4 G M}{r c_{o}^{2}}\right)^{1 / 4}
$$

If we consider $v_{o}$ the frequency of a photon outside the gravitational field, since $m_{p h_{o}} c_{o}^{2}=h v_{o}$, the correlation between $v$ and $v_{o}$, which is the change in frequency when a photon enters a gravitational field, is given by the relation:

$$
v=v_{o}\left(1-\frac{4 G M}{r c_{o}^{2}}\right)^{1 / 4}
$$

There is yet another change that the elementary photonic wave undergoes, that is, the photon, and that is the change in wavelength. To determine this change we will use the equation $c=v \lambda$. According to Equations (10) and (18), we get :

$$
\lambda=\frac{c}{v}=\lambda_{o}\left(1-\frac{4 G M}{r c_{o}^{2}}\right)^{1 / 4}
$$

where $\lambda_{o}=c_{o} / v_{o}$. According to the hypothesis of the absolute reference system, the bound photons, which are the building elements of the elementary particles of matter, will undergo these changes in frequency and wavelength. If the closed orbit of a bound photon has a length equal to $n \lambda$, where $n=1,2, \cdots$, then the time of a period is $T=n \lambda / c$, while, outside the gravitational field, the corresponding time is $T_{o}=n \lambda_{o} / c_{o}$. Therefore:

$$
T=T_{o} \frac{c_{o} \lambda}{c \lambda_{o}}=T_{o}\left(1-\frac{4 G M}{r c_{o}^{2}}\right)^{-1 / 4}
$$

According to the hypothesis of the absolute reference system, the estimated time is inversely proportional to the time of the aforementioned period ([1], 2.4.1. Contraction of Length and Time, pp. 440-441). Assuming that $t_{g}$ is the estimated time recorded between two events occurring inside the gravitational field using a clock at a fixed position $\boldsymbol{r}$ also inside the gravitational field, and $t$ 
is the corresponding estimated time using a clock outside the gravitational field, then the correlation of these times is given by the equation:

$$
\frac{t_{g}}{t}=\frac{T_{o}}{T} \Leftrightarrow t_{g}=t\left(1-\frac{4 G M}{r c_{o}^{2}}\right)^{1 / 4} \simeq t\left(1-\frac{G M}{r c_{o}^{2}}\right)
$$

The corresponding result of the general theory of relativity is:

$$
t_{g}=t\left(1-\frac{2 G M}{r c_{o}^{2}}\right)^{1 / 2} \simeq t\left(1-\frac{G M}{r c_{o}^{2}}\right)
$$

Therefore the estimated time, based on the hypothesis of the absolute reference system, with a very good approximation is the same as that of the general theory of relativity. This estimated time value has been experimentally confirmed ([5] [6]). Also this change in the estimation of time in the gravitational field affects all estimated values of speed, momentum, force, energy and frequency. It certainly does not affect the mass and wavelength, which are basic (fundamental) quantities.

Suppose that using a clock within the gravitational field, the estimated oscillation period of a photon is $T_{p h_{g}}$, while the estimated oscillation period of the same photon using a clock outside the gravitational field is $T_{p h}$, then, according to the relation (21), we get the equation:

$$
T_{p h_{g}}=T_{p h}\left(1-\frac{4 G M}{r c_{o}^{2}}\right)^{1 / 4}
$$

therefore, since $v=1 / T_{p h}$, and assuming that $v_{g}$ is the estimated frequency of an oscillator within the gravitational field, according to the relation (18) the following equality arises:

$$
v_{g}=\frac{1}{T_{p h_{g}}}=v\left(1-\frac{4 G M}{r c_{o}^{2}}\right)^{-1 / 4}=v_{o}
$$

where $v_{o}$ is the frequency of the photon when it is outside the gravitational field. Therefore the estimated frequency appears to be constant and equal to the frequency $v_{o}$ as the photon enters or leaves the gravitational field.

The estimated velocity $c_{g}=\mathrm{dl} / \mathrm{d} t_{g}$ of the photon, since $\mathrm{d} t_{g}=\mathrm{d} t\left(1-4 G M /\left(r c_{o}^{2}\right)\right)^{1 / 4}, c=\mathrm{d} l / \mathrm{d} t$, and $\mathrm{d} l=|\mathrm{d} r|=\sqrt{\mathrm{d} r^{2}+r^{2} \mathrm{~d} \theta^{2}}$, is given by the relation:

$$
\begin{aligned}
c_{g} & =c\left(1-\frac{4 G M}{r c_{o}^{2}}\right)^{-1 / 4} \\
& =c_{o}\left(1-\frac{4 G M}{r c_{o}^{2}}\right)^{1 / 2}\left(1-\frac{4 G M}{r c_{o}^{2}}\right)^{-1 / 4} \\
& =c_{o}\left(1-\frac{4 G M}{r c_{o}^{2}}\right)^{1 / 4}
\end{aligned}
$$

so, according to the relations (11) and (24), the estimated energy of the photon is 
given by the relation:

$$
\mathcal{E}_{p h_{g}}=m_{p h} c_{g}^{2}=m_{p h_{o}} c_{o}^{2}\left(1-\frac{4 G M}{r c_{o}^{2}}\right)^{-1 / 4}
$$

Therefore, in a first order approach, the estimated energy of the photon becomes:

$$
\mathcal{E}_{p h_{g}} \simeq m_{p h_{o}} c_{o}^{2}+\frac{G M m_{p h_{o}}}{r}
$$

and since $m_{p h_{o}} c_{o}^{2}=h v_{o}=h v_{g}$, the following relation arises:

$$
\mathcal{E}_{p h_{g}} \simeq h v_{g}+\frac{G M m_{p h_{o}}}{r}
$$

The conclusion we draw on the basis of the relation (27), is that the estimated value of the photonic frequency is not enough to estimate the photon energy, since the term $G M m_{p h_{o}} / r$, which is the absolute value of the dynamic energy, is added. This also means that the term $G M m_{p h_{o}} / r$ is always added to the amount of energy $h v_{g}$ when estimating the photonic energy of any photon that is emitted or propagated in the gravitational field.

Let us consider the example of a hydrogen atom located at a position $r$ within the gravitational field. This atom is considered as a physical system, where the electron moves under the influence of the electric field of the nucleus. According to the hypothesis of an absolute reference system, the mass of an electron outside the gravitational field is given by the equation:

$$
m_{o}=\sum_{i=1}^{N} m_{p h_{i}}
$$

where $m_{p h_{i}}$ is the mass of the $i$ bound photon and $N$ is the number of bound photons. Therefore based on the relation $h v_{c_{i}}=m_{p h_{i}} c^{2}$, where $v_{c_{i}}$ is the mass frequency of the $i$ bound photon, we get the equation:

$$
m_{o} c_{o}^{2}=h \sum_{i=1}^{N} v_{c_{i}}
$$

which is equivalent to Equation (2.1) in [2] (2.1. Particle-Frequency and Wavelength, p. 752). Within the gravitational field an amount of energy equal to $G M m_{p h_{i}} / r$ is added to each term $h v_{c_{i}}$ of the sum of Equation (28). Therefore, a constant amount of energy is added to electron energy (at a fixed position $\boldsymbol{r}$ ), given by the equation:

$$
E_{g}=\frac{G M}{r} \sum_{i=1}^{N} m_{p h_{i}}=\frac{G M m_{o}}{r}
$$

However, this constant amount of energy can not affect the energy values that have come from transitions of the aforementioned atom in our example. This means that the differences between the energy stations of the hydrogen atom within the gravitational field will be equal to the corresponding differences of the same atom outside the gravitational field. But there is a difference. The photons that have resulted from these transitions inside the gravitational field will have a lower 
frequency than the corresponding ones outside the gravitational field, i.e. they will have shifted to red, due to the existence of the term $G M m_{p h_{o}} / r$ in the relation (27). Because the estimated photonic energy, $\mathcal{E}_{p h_{g}}$, at the position $\boldsymbol{r}$ resulting from an energy transition is equal to the corresponding photon energy outside the gravitational field, this redshift is not perceived. However, this redshift is perceived when a photon, which has come from the aforementioned energy transition inside the gravitational field at position $\boldsymbol{r}$, moves radially towards the exit from the gravitational field, since the estimated energy $h v_{g}+G M m_{p h} / r$ decreases as the radial distance $r$ increases, and the estimated frequency $v_{g}$, already shifted to red, remains constant.

The calculation of the estimated energy as a function of wavelength, according to the relations (19) and (25), given that $m_{p h_{o}} c_{o}^{2}=h v_{o}=h c_{o} / \lambda_{o}$, gives us the relation:

$$
\mathcal{E}_{p h_{g}}=h \frac{C_{o}}{\lambda}
$$

while for a photon outside the gravitational field (for $r \rightarrow \infty$ ), the estimated energy is $\mathcal{E}_{p h_{o}}=h c_{o} / \lambda_{o}$. The change in the estimated energy of a photon entering the gravitational field is given by the relation:

$$
\Delta \mathcal{E}_{p h_{g}}=\mathcal{E}_{p h_{g}}-\mathcal{E}_{p h_{o}}=h c_{o}\left(\frac{1}{\lambda}-\frac{1}{\lambda_{o}}\right)
$$

while the relative change in the estimated energy, which gives us a quantitative estimate of the shift to red, is given by the equation:

$$
\frac{\Delta \mathcal{E}_{p h_{g}}}{\mathcal{E}_{p h_{o}}}=\frac{\lambda \lambda_{o}-\lambda}{\lambda}=\frac{\Delta \lambda}{\lambda}
$$

If we define the estimated gravitational redshift as $v_{\mathrm{GRS}}=c_{0} \Delta \lambda / \lambda$, then this estimate is expressed by the following relation:

$$
v_{\mathrm{GRS}}=c_{o} \frac{\Delta \lambda}{\lambda}=c_{o}\left[\left(1-\frac{4 G M}{r c_{o}^{2}}\right)^{-1 / 4}-1\right] \simeq \frac{G M}{r c_{o}}
$$

The corresponding estimate according to the general theory of relativity is given by the relation:

$$
v_{\mathrm{GRS}}=c_{o} \frac{\Delta \lambda}{\lambda}=c_{o}\left[\left(1-\frac{2 G M}{r c_{o}^{2}}\right)^{-1 / 2}-1\right] \simeq \frac{G M}{r c_{o}}
$$

\section{Using the Experimental Tests of the General Theory of Relativity to Experimentally Test the Hypothesis of an Absolute Reference System}

In this section we examine the known experimental tests of the general theory of relativity, in order to determine whether the experimental results confirm the hypothesis of the absolute reference system.

In order to achieve this we use the theoretical study that has already been de- 
veloped in the previous three sections.

\subsection{The Advance of the Perihelion of Mercury}

Denoting the mass of the Sun by $M$ and the mass of Mercury by $m$, according to Equations (12) and (15), we get the equation:

$$
\frac{\mathrm{d}^{2} u}{\mathrm{~d} \theta^{2}}+u=\frac{G M m_{o}^{2}}{L^{2}}\left(1-\frac{4 G M}{r c_{o}^{2}}\right)^{-3 / 2}
$$

Given that $u=1 / r$, and $\left(1-4 G M /\left(r c_{o}^{2}\right)\right)^{-3 / 2} \simeq 1+6 G M /\left(r c_{o}^{2}\right)$, the first order approach is given by the differential equation:

$$
\frac{\mathrm{d}^{2} u}{\mathrm{~d} \theta^{2}}+\left(1-\frac{6 G^{2} M^{2} m_{o}^{2}}{c_{o}^{2} L^{2}}\right) u=\frac{G M m_{o}^{2}}{L^{2}}
$$

We define the constants:

$$
\begin{gathered}
A=1-\frac{6 G^{2} M^{2} m_{o}^{2}}{c_{o}^{2} L^{2}} \\
B=\frac{G M m_{o}^{2}}{L^{2}}
\end{gathered}
$$

The solution of the differential Equation (36) has the form:

$$
u=\frac{1}{r}=\frac{B}{A}+K \cos (\sqrt{A} \theta)
$$

where $K$ is constant. Because the orbit of Mercury around the Sun is elliptical it will obey the relation:

$$
\frac{1}{r}=\frac{1}{a\left(1-\varepsilon^{2}\right)}+\frac{\varepsilon}{a\left(1-\varepsilon^{2}\right)} \cos \Phi
$$

where $a$ is the length of the semi-major axis of the elliptical orbit and $\varepsilon$ is the eccentricity. As is well known the eccentricity of the elliptical orbit is less than unit. Comparing Equations (37) and (38), if we consider:

$$
\varepsilon^{2}=\frac{6 G^{2} M^{2} m_{o}^{2}}{c_{o}^{2} L^{2}}
$$

then $A=1-\varepsilon^{2}$ and $B=1 / a$. The minimum possible distance, $R_{\pi}$, of Mercury from the sun occurs when the $1 / r$ becomes maximum, and this happens when the $\cos \Phi$ in Equation (38) becomes equal to unit, in which case the following equation is obtained:

$$
\frac{1}{R_{\pi}}=\frac{1}{a(1-\varepsilon)}
$$

whereas respectively when $\cos (\sqrt{A} \theta)=1$, by Equation (37) we get:

$$
K=\frac{1}{R_{\pi}}-\frac{B}{A}
$$

Indeed this value of $K$ agrees with the factor which is multiplied by $\cos \Phi$ in Equation (38), since: 


$$
K=\frac{1}{R_{\pi}}-\frac{B}{A}=\frac{1}{a(1-\varepsilon)}-\frac{1}{a\left(1-\varepsilon^{2}\right)}=\frac{\varepsilon}{a\left(1-\varepsilon^{2}\right)}
$$

Two consecutive minimizations of the distance $r$ are performed for $\sqrt{A} \theta=0$ and for $\sqrt{A} \theta=2 \pi$. For $\sqrt{A} \theta=0$ the angle $\theta$ is zero, while for $\sqrt{A} \theta=2 \pi$ the angle $\theta$ is given by the relation:

$$
\theta=\frac{2 \pi}{\sqrt{A}} \simeq 2 \pi+\frac{6 \pi G^{2} M^{2} m_{o}^{2}}{c_{o}^{2} L^{2}}
$$

This result is the same as that of the general theory of relativity ([7], $₫ 101$. Motion in a centrally symmetric gravitational field) and is confirmed by observations already announced in the 19th century.

The contributions to the motion of the perihelia of Mercury and the earth are described in [8] (see [8], TABLE II). A table, similar to the table by Clemence (1947), with significant improvements in accuracy and including additional effects is in [9] (see [9], TABLE III).

A similar description, based on the concept of "ether disturbance" due to the gravitational field and giving similar results, has been announced by Antonis $\mathrm{N}$. Agathangelidis, in [10]. However, this description is different from this of the hypothesis of the absolute reference system.

\subsection{The Deflection of Light in the Gravitational Field of the Sun}

When a photon enters the gravitational field of the sun, its energy changes from the value $m_{p h_{o}} c_{o}^{2}$ to the value $m_{p h} c^{2}$, when it leaves the gravitational field, it takes again the value $m_{p h_{o}} c_{o}^{2}$. Also the motion of said photon at a great distance from the sun, where it can be considered to be outside the gravitational field, tends to be asymptotically a straight line, while inside the gravitational field it is curvilinear, due to the effect of the gravitational force of the Sun according to the relation (4). Since the force is central, the trajectory equation has the geometric shape of the hyperbola, so, in polar coordinates $r, \Phi$, obeys the equation:

$$
\frac{1}{r}=\frac{1}{a\left(\varepsilon^{2}-1\right)}+\frac{\varepsilon}{a\left(\varepsilon^{2}-1\right)} \cos \Phi
$$

where $\varepsilon$ is the eccentricity, which is greater than unit, and $a$ is the distance from the center $C$ to the vertex $V$ called the semi-major axis (Figure 1 ).

The solution of the differential Equation (16) is given by the equation:

$$
u=\frac{1}{r}=\frac{B^{\prime}}{A^{\prime}}+K^{\prime} \cos \left(\sqrt{A^{\prime}} \theta\right)
$$

where $A^{\prime}, B^{\prime}$ are the constants given by the equations:

$$
\begin{gathered}
A^{\prime}=1-\frac{6 G^{2} M^{2} m_{p h_{o}}^{2}}{c_{o}^{2} L^{2}} \\
B^{\prime}=\frac{G M m_{p h_{o}}^{2}}{L^{2}}
\end{gathered}
$$




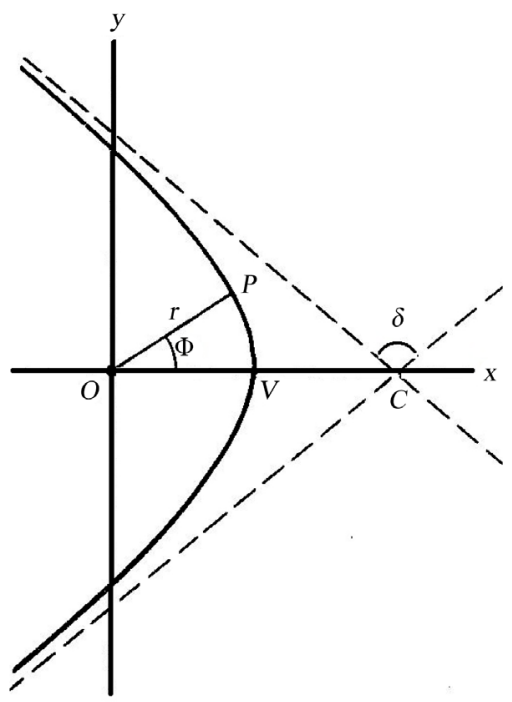

Figure 1. The continuous curved line is the elliptical orbit of the photon within a gravitational field. The hyperbolic orbit asymptotically approaches the dashed lines which are the directions of motion of the photon at a great distance from the spherical body of mass $M$. The angle $\delta$ is the angle of deflection of the photon.

Comparing Equations (42) and (43), we observe that by setting:

$$
\varepsilon^{2}=\frac{c_{o}^{2} L^{2}}{6 G^{2} M^{2} m_{p h_{o}}^{2}}
$$

then $\varepsilon>1$, and we take the equation:

$$
A^{\prime}=\frac{\varepsilon^{2}-1}{\varepsilon^{2}}
$$

and in order to be $B^{\prime} / A^{\prime}=1 /\left(a\left(\varepsilon^{2}-1\right)\right)$ the following equality must be valid:

$$
B^{\prime}=\frac{1}{a \varepsilon^{2}}
$$

The minimum distance, $R_{\varepsilon}$, is calculated using the relation (42), for $\cos \Phi=1$ where $\Phi=0$, so we get the equation:

$$
R_{\varepsilon}=a(\varepsilon-1)
$$

The value of $R_{\varepsilon}$ is equal to the length $O V$ of Figure 1. The corresponding value of $R_{\varepsilon}$ is given by Equation (43) for $\cos \left(\sqrt{A^{\prime}} \theta\right)=1$, so we get the equation:

$$
\frac{1}{R_{\varepsilon}}=\frac{B^{\prime}}{A^{\prime}}+K^{\prime}
$$

Substituting the $A^{\prime}, B^{\prime}$ and $R_{\varepsilon}$, the value of $K^{\prime}$ is given by the equation:

$$
K^{\prime}=\frac{1}{R_{\varepsilon}}-\frac{B^{\prime}}{A^{\prime}}=\frac{\varepsilon}{a\left(\varepsilon^{2}-1\right)}
$$

which is the factor that is multiplied by $\cos \Phi$ in Equation (42). Therefore 
$\Phi=\sqrt{A^{\prime}} \theta$. The maximum angle, $\Phi_{\max }$, is obtained when $r$ becomes infinite, so that the direction of $O P$ becomes parallel to the dashed asymptotic line (Figure 1). In this case Equation (42) gives:

$$
\Phi_{\max }=\lim _{r \rightarrow \infty} \cos \Phi=-\frac{1}{\varepsilon}
$$

Therefore the deflection angle $\delta$, according to Figure 1 , is given by the equation:

$$
\delta=2 \Phi_{\max }-\pi
$$

so:

$$
\cos \Phi_{\max }=\cos \left(\frac{\pi}{2}+\frac{\delta}{2}\right)=-\sin \left(\frac{\delta}{2}\right)
$$

Since, as we will see below, the angle $\delta$ is very small, with the help of the relation (45) we can consider that:

$$
-\sin \left(\frac{\delta}{2}\right) \simeq-\frac{\delta}{2} \Leftrightarrow \delta \simeq \frac{2}{\varepsilon}
$$

The corresponding angle $\delta^{\prime}$ that can be derived from the angle $\theta$ according to the relation $\theta_{\max }=\Phi_{\max } / \sqrt{A^{\prime}}$, where $\theta_{\max }=\left(\pi+\delta^{\prime}\right) / 2$ and $\Phi_{\text {max }}=(\pi+\delta) / 2$, is given by the following relation:

$$
\delta^{\prime}=\frac{\delta}{\sqrt{A^{\prime}}} \simeq \frac{2}{\varepsilon}\left(1-\frac{1}{\varepsilon^{2}}\right)^{-1 / 2} \simeq \frac{2}{\varepsilon}+\frac{1}{\varepsilon^{3}} \simeq \frac{2}{\varepsilon}
$$

which means that the angle $\delta^{\prime}$, with a very good approximation, does not differ from the angle $\delta$. We can determine the value of $\varepsilon$ from Equation (44), having previously determined the value of the constant angular momentum. The angular momentum can be calculated at the position of the minimum distance, $R_{\varepsilon}$, ie when the photon passes through the position $V$ of Figure 1, so it is given by the relation:

$$
\begin{aligned}
L & =m_{\mathrm{V}} c_{\mathrm{V}} R_{\varepsilon}=m_{p h_{o}}\left(1-\frac{4 G M}{R_{\varepsilon} c_{o}^{2}}\right)^{-3 / 4} c_{o}\left(1-\frac{4 G M}{R_{\varepsilon} c_{o}^{2}}\right)^{1 / 2} R_{\varepsilon} \\
& \simeq m_{p h_{o}} c_{o}\left(R_{\varepsilon}+\frac{G M}{c_{o}^{2}}\right)
\end{aligned}
$$

where $m_{\mathrm{V}}$ and $c_{\mathrm{V}}$ is the photon mass and velocity when the photon passes through the position of the minimum distance $R_{\varepsilon}$. If we set $R_{\varepsilon}^{\prime}=R_{\varepsilon}+G M / c_{o}^{2}$, the deflection angle is given by the equation:

$$
\delta=\frac{2}{\varepsilon}=\frac{2 \sqrt{6} G M}{R_{\varepsilon}^{\prime} c_{o}^{2}}
$$

If we choose the distance $R_{\varepsilon}$ to be equal to the radius of the Sun, and the mass $M$ equal to the mass of the Sun, then $G M / c_{o}^{2}=1.47 \mathrm{~km}$, so we get the result :

$$
\delta \simeq 2^{\prime \prime} .13
$$


The corresponding value derived from the general theory of relativity is:

$$
\delta_{\mathrm{GR}}=\frac{4 G M}{R_{\varepsilon} c_{o}^{2}} \simeq 1^{\prime \prime} .75
$$

The result of calculating the deviation angle based on the hypothesis of the absolute reference system, which is $2 " .13$, is closer to the experimental value than the result of the general theory of relativity. This experimental value was obtained from measurements at the Sobral in 1919 ([11], V. General Conclusions. p. 330), which were:

From declinations 1".94;

From right ascensions $2 " .06$.

The result from declinations is about twice the weight of that from right ascensions, so that the mean result is $1 " .98$.

As stated in the article [11], "it was recognized that these would prove to be much the most trustworthy", in contrast to the measurement in Principe, for which the same article states that "The Principe observations were generally interfered with by cloud...”. The experimental results are presented in Table 1. More details are mentioned in a relevant lecture, about gravitational lensing, in [12].

Bergmann's book ([13], The deflection of light in a Schwarzschild field $\mathrm{p}$. 221) states that "The predicted deflection amounts to no more than about 1.75 " and is just outside the limits of experimental error. A quantitative agreement between the predicted and the observed effects cannot be regarded as significant".

Table 1. General conclusions.

\begin{tabular}{|c|c|c|}
\hline Obtained with: & Results: & Remarks: \\
\hline $\begin{array}{l}\text { The 4-inch } \\
\text { lens at Sobral }\end{array}$ & $\begin{array}{l}\text { From declinations } 1 " .94 \\
\text { From right ascensions } 2 " .06 \\
\text { the mean result is } 1 " .98 \\
\text { probable error of about } \pm 0 " .12 \\
\text { (The result from declinations } \\
\text { is about twice the weight } \\
\text { of that from right ascensions) }\end{array}$ & $\begin{array}{l}\text { In summarizing the results of the two } \\
\text { expeditions, the greatest weight must be } \\
\text { attached to those obtained with the 4-inch } \\
\text { lens at Sobral. From the superiority of the } \\
\text { images and the larger scale of the } \\
\text { photographs it was recognized that these } \\
\text { would prove to be much the most } \\
\text { trustworthy. Further, the agreement of the } \\
\text { results derived independently from the right } \\
\text { ascensions and declinations, and the } \\
\text { accordance of the residuals of the individual } \\
\text { stars ([11], p. 308) provides a more } \\
\text { satisfactory check on the results than was } \\
\text { possible for the other instruments. }\end{array}$ \\
\hline $\begin{array}{c}\text { Principe } \\
\text { observations }\end{array}$ & $\begin{array}{l}\text { The deflection obtained } \\
\text { was } 1 " .61 \\
\text { probable error of } \\
\text { about } \pm 0 " .30\end{array}$ & $\begin{array}{l}\text { The Principe observations were generally } \\
\text { interfered with by cloud. The unfavorable } \\
\text { circumstances were perhaps partly } \\
\text { compensated by the advantage of the } \\
\text { extremely uniform temperature of the island. }\end{array}$ \\
\hline $\begin{array}{l}\text { The Sobral } \\
\text { astrographic } \\
\text { plates }\end{array}$ & $\begin{array}{l}\text { The deflection obtained } \\
\text { was } 0 \text { ".93 } \\
\text { discordant by an amount } \\
\text { much beyond the limits } \\
\text { of its accidental error }\end{array}$ & $\begin{array}{l}\text { For the reasons already described } \\
\text { at length not much weight is attached to } \\
\text { this determination. }\end{array}$ \\
\hline
\end{tabular}




\subsection{The Time Delay of Light}

We will now study the test proposed by Irwin I. Shapiro in order to measure a time delay (Shapiro delay) in the round-trip travel time for radar signals reflecting off other planets ([14]), sometimes called the fourth "classical test" of general relativity.

We consider that an electromagnetic signal is emitted from Earth, reflected on a planet (or spacecraft) and returned to Earth, but under the influence of the Sun's gravitational field during its motion towards the planet and during its return to the Earth. We will calculate the time delay due to the effect of the solar gravitational field on an elementary photonic signal, i.e. a photon. The path of the examined photon is the path $C B A B C$, of the axis $x$ shown in Figure 2. Of course the deflection angle, which was calculated in the previous section, is very small, so the above-mentioned orbit is considered straight. Here we have ignored the motion of the Earth and planets during the round trip of the signal, because the corresponding velocities, estimated in the reference frame of the solar system, are much slower than the speed of light in vacuum.

During the path $\mathrm{CB}$, i.e. after the signal leaves the Earth, when the photon is in a random position $\mathrm{P}$ (Figure 2), as distance $x$ we consider the length of the segment $\mathrm{BP}$, and $r$ is the distance of point $\mathrm{P}$ from the center of the Sun. The length of $d$ is the minimum possible distance of the photon from the center of the Sun during its round trip path. As the photon moves from the position $\mathrm{C}$ to the position B, that is, immediately after its departure from Earth, the distance $x$ decreases, so the speed of the photon, $c$, which depends on the radial distance $r$, will be $c=-\mathrm{d} x / \mathrm{d} t$. Therefore, according to the relation (10), the time differential is given by the relation:

$$
\mathrm{d} t=-\frac{\mathrm{d} x}{c}=-\frac{1}{c_{o}}\left(1-\frac{4 G M}{r c_{o}^{2}}\right)^{-1 / 2} \mathrm{~d} x
$$

A first order approximation of time differential is given by the equation:

$$
\mathrm{d} t=-\frac{\mathrm{d} x}{c}=-\frac{1}{c_{o}}\left(1+\frac{2 G M}{r c_{o}^{2}}\right) \mathrm{d} x
$$

Because $x=\sqrt{r^{2}-d^{2}}$ the differential $\mathrm{d} x$ is given by the relation $\mathrm{d} x=r \mathrm{~d} r / \sqrt{r^{2}-d^{2}}$, and $r_{e}$ is the distance of the Earth from the Sun, the integral from position $\mathrm{C}$ to position $\mathrm{B}$ gives us:

$$
\Delta t_{\mathrm{CB}}=-\int_{r_{e}}^{d} \frac{1}{c_{o}}\left(1+\frac{2 G M}{r c_{o}^{2}}\right) \frac{r \mathrm{~d} r}{\sqrt{r^{2}-d^{2}}}=\frac{x_{e}}{c_{o}}+\frac{2 G M}{c_{o}^{3}} \ln \left(\frac{r_{e}+x_{e}}{d}\right)
$$

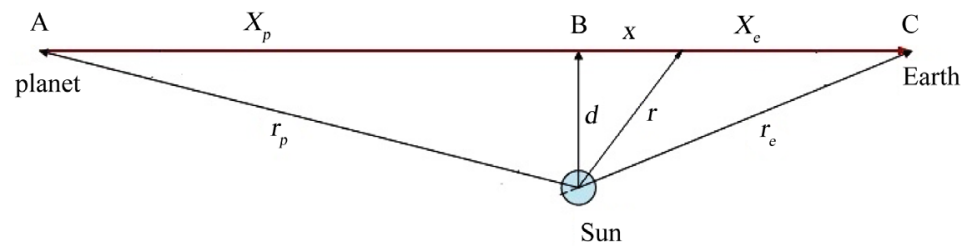

Figure 2. Geometry of light deflection measurements. 
where $x_{e}=\sqrt{r_{e}^{2}-d^{2}}$, i.e. the distance $x_{e}$ is the distance CB of Figure 2. As the photon moves from position $\mathrm{B}$ to position $A$, the distance $x$ increases, so the travel time interval BA is given by the relation:

$$
\Delta t_{\mathrm{BA}}=\int_{d}^{r_{p}} \frac{1}{c_{o}}\left(1+\frac{2 G M}{r c_{o}^{2}}\right) \frac{r \mathrm{~d} r}{\sqrt{r^{2}-d^{2}}}=\frac{x_{p}}{c_{o}}+\frac{2 G M}{c_{o}^{3}} \ln \left(\frac{r_{p}+x_{p}}{d}\right)
$$

The time of travel from Earth to the planet is given by the relation:

$$
\Delta t_{\mathrm{CA}}=\Delta t_{\mathrm{CB}}+\Delta t_{\mathrm{BA}}=\frac{x_{e}+x_{p}}{c_{o}}+\frac{2 G M}{c_{o}^{3}} \ln \left(\frac{\left(r_{e}+x_{e}\right)\left(r_{p}+x_{p}\right)}{d^{2}}\right)
$$

Therefore the total time of the round trip path is calculated according to the relation:

$$
\Delta t=\frac{2\left(x_{e}+x_{p}\right)}{c_{o}}+\frac{4 G M}{c_{o}^{3}} \ln \left(\frac{\left(r_{e}+x_{e}\right)\left(r_{p}+x_{p}\right)}{d^{2}}\right)
$$

This result agrees with the corresponding result of the general theory of relativity ([15], 7.2 The Time Delay of Light, Equation (7.31)). Specifically the time period $\Delta t$, according to the general theory of relativity, is given by the relation:

$$
\Delta t=\frac{2\left(x_{e}+x_{p}\right)}{c_{o}}+2(1+\gamma) \frac{G M}{c_{o}^{3}} \ln \left(\frac{\left(r_{e}+x_{e}\right)\left(r_{p}+x_{p}\right)}{d^{2}}\right)
$$

where $\gamma=1$. According to Newtonian physics, this time interval is calculated according to the relation $\Delta t_{\mathrm{N}}=2\left(x_{e}+x_{p}\right) / c_{o}$. The additional "time delay" produced by the second term in Equation (55) is a maximum when the planet is on the far side of the Sun from the Earth (superior conjunction), i.e., when

$$
x_{e} \simeq r_{e}, \quad x_{p} \simeq r_{p}, \quad d \simeq \text { solar radius }
$$

then, the additional time delay of light, due exclusively to the effect of the gravitational field of the Sun, is given by the equation:

$$
\Delta t_{\mathrm{G}}=\frac{4 G M}{c_{o}^{3}} \ln \left(\frac{4 r_{e} r_{p}}{d^{2}}\right)
$$

If we denote by $R_{s}$ the radius of the Sun, then Equation (57) give us:

$$
\begin{aligned}
\Delta t_{\mathrm{G}} & =-\frac{4 G M}{c_{o}^{3}} \ln \left[\left(\frac{d}{R_{s}}\right)^{2}\left(\frac{r_{e}}{r_{p}}\right)\left(\frac{R_{s}}{2 r_{e}}\right)^{2}\right] \\
& =\frac{4 G M}{c_{o}^{3}}\left\{2 \ln \left(\frac{2 r_{e}}{R_{s}}\right)-\ln \left[\left(\frac{d}{R_{s}}\right)^{2}\left(\frac{r_{e}}{r_{p}}\right)\right]\right\}
\end{aligned}
$$

Since the distance from Earth to the Sun is equal to the astronomical unit, that is $r_{e}=A U$, and the Solar radius is equal to $0.005 A U$, it follows that $2 \ln \left(2 r_{e} / R_{s}\right) \simeq 12$. Also, because $2 G M / c_{o}^{2} \simeq 3 \mathrm{~km}$, it follows that $4 G M / c_{o}^{3} \simeq 20 \mu$ s. Therefore the relation (58) becomes:

$$
\Delta t_{\mathrm{G}}=240 \mu \mathrm{s}-\ln \left[\left(\frac{d}{R_{s}}\right)^{2}\left(\frac{A U}{r_{p}}\right)\right] \times 20 \mu \mathrm{s}
$$


which is the relativistic prediction ([15], 7.2 The Time Delay of Light, Equation (7.33)). Many relative measurements to confirm the relativistic equality $\gamma=1$ have been made since the early 1970s for more than three decades ([16] [17] [18]).

As stated in Will's book ([15], 7.2 The Time Delay of Light, p. 174) "Since one does not have access to a "Newtonian" signal against which to compare the round trip travel time of the observed signal, it is necessary to do a differential measurement of the variations in round trip travel times as the target passes through superior conjunction, and to look for the logarithmic behavior". An important relevant experiment was reported in 2003, in [19], from Doppler tracking of the Cassini spacecraft while it was on its way to Saturn, with a result $\gamma-1=(2.1 \pm 2.3) \times 10^{-5}$.

\subsection{The Gravitational Redshift}

We will first study the redshift due to the Earth's gravitational field for example, which is observed if two measurements of the estimated energy of a photon are taken at two different radial distances $r_{1}$ and $r_{2}$ that differ only slightly, and $r_{1}<r_{2}$. Also we consider that $r_{1}$ is equal to the distance of the earth ground of the experiment from the center of the Earth. The ratio of the two estimated energies, determined by the relation (25), is calculated as follows:

$$
\frac{\mathcal{E}_{p h_{g}}\left(r_{2}\right)}{\mathcal{E}_{p h_{g}}\left(r_{1}\right)}=\frac{m_{p h_{o}} c_{o}^{2}\left(1-\frac{4 G M}{r_{2} c_{o}^{2}}\right)^{-1 / 4}}{m_{p h_{o}} c_{o}^{2}\left(1-\frac{4 G M}{r_{1} c_{o}^{2}}\right)^{-1 / 4}} \simeq\left(1-\frac{G M}{c_{o}^{2}} \frac{r_{1}-r_{2}}{r_{1} r_{2}}\right)
$$

If $h=r_{2}-r_{1}$ is the height, $R$ is the radius of the Earth, and $g=G M / R^{2}$ then the previous relation becomes:

$$
\frac{\mathcal{E}_{p h_{g}}\left(r_{2}\right)}{\mathcal{E}_{p h_{g}}\left(r_{1}\right)} \simeq 1-\frac{g h}{c_{o}^{2}}
$$

This result agrees with the corresponding prediction of the general theory of relativity and has been experimentally confirmed ([20]).

The gravitational redshift in the solar photosphere obeys the relation:

$$
v_{\mathrm{GRS}, \odot}=c_{o} \frac{\Delta \lambda_{s}}{\lambda_{s}}
$$

where $\Delta \lambda_{s}=\lambda_{o}-\lambda_{s}$, and $\lambda_{s}$ is the wavelength in the solar photosphere. If $R_{\odot}$ is the radius of the sun, then, according to Equation (33), the relation (62) becomes:

$$
v_{\mathrm{GRS}, \odot} \simeq \frac{G M}{c_{o} R_{\odot}}
$$

This result is also predicted by the general theory of relativity. The same effect from the solar photosphere to $1 A U$ amounts to: 


$$
v_{\mathrm{GRS}, \odot, 1 \mathrm{AU}}=\frac{G M}{c_{o}}\left(\frac{1}{R_{\odot}}-\frac{1}{A U}\right)=633.35 \mathrm{~m} \cdot \mathrm{s}^{-1}
$$

A related experiment confirming this result (in [21]) was conducted recently, in 2020, and a team of scientists published the most accurate measurement of the solar gravitational redshift so far, made by analyzing Fe spectral lines in sunlight reflected by the moon. Their measurement of a mean global $638 \pm 6 \mathrm{~m} / \mathrm{s} \mathrm{li}$ neshift is in agreement with the theoretical value of $633.1 \mathrm{~m} / \mathrm{s}$.

A lot of terrestrial and astronomical observations confirm these results. Also the theoretical study of the redshift due to the gravitational field based on the hypothesis of the absolute reference system provides solutions to relevant divergent views that appear in the literature, for example regarding the problem "Is there a physical process causing the redshift?" in [22].

\section{Conclusion}

The physics of an absolute reference system is a comprehensive and self-contained view of physical reality, extending to all areas of the physical sciences, and confirmed by a wide range of scientific observations and experiments, including all experiments performed from time to time in order to confirm the special and general theory of relativity. It is found that some of these experiments do not confirm the theory of relativity, but nevertheless confirm the hypothesis of the absolute reference system. In this article, the calculations are confirmed by the experimental results, and it is also found that the prediction for the experiment on the deflection of light in the gravitational field of the Sun on the basis of the hypothesis of the absolute reference system, is in better agreement with the experimental data compared to the corresponding prediction of the general theory of relativity. It is therefore necessary to carry out an experiment in order to take high-precision measurements of light deflection, in the special case of the effect of the gravitational field of the Sun.

\section{Conflicts of Interest}

The author declares no conflicts of interest regarding the publication of this paper.

\section{References}

[1] Patrinos, K. (2019) The Physics of an Absolute Reference System. Journal of Applied Mathematics and Physics, 7, 431-475. https://doi.org/10.4236/jamp.2019.73033

[2] Patrinos, K. (2019) Classical Quantum Field Theory Based on the Hypothesis of the Absolute Reference System. Journal of Applied Mathematics and Physics, 7, 747-780. https://doi.org/10.4236/jamp.2019.74052

[3] Patrinos, K. (2020) The Confirmation of Hypothesis of the Absolute Reference System. Journal of Applied Mathematics and Physics, 8, 999-1015. https://doi.org/10.4236/jamp.2020.86078

[4] Goldstein, H. (1980) Classical Mechanics. 2nd Edition, Addison-Wesley Publishing 
Company, Inc., Boston.

[5] Hafele, J.C. and Keating, R.E. (1972) Around-the-World Atomic Clocks: Predicted Relativistic Time Gains. Science, 177, 166-168.

https://doi.org/10.1126/science.177.4044.166

[6] Hafele, J.C. and Keating, R.E. (1972) Around-the-World Atomic Clocks: Observed Relativistic Time Gains. Science, 177, 168-170. https://doi.org/10.1126/science.177.4044.168

[7] Landau, L. and Lifshitz, E. (1975) The Classical Theory of Fields. Pergamon Press Ltd., Oxford. (Copyright 1975)

[8] Clemence, G.M. (1947) The Relativity Effect in Planetary Motions. Reviews of Modern Physics, 19, 361-364. https://doi.org/10.1103/RevModPhys.19.361

[9] Park, R.S., Folkner, W.M., Konopliv, A.S., Williams, J.G., Smith, D.E. and Zuber, M.T. (2017) Precession of Mercury's Perihelion from Ranging to the MESSENGER Spacecraft. The Astronomical Journal, 153, Article No. 121. https://doi.org/10.3847/1538-3881/aa5be2

[10] Agathangelidis, N.A. (2011) Relativity Replaced-Ether Found around Earth: The Ether of Stokes Rules Pseudo-Relativistic Physics. LAP Lambert Academic Publishing, Chisinau.

[11] Dyson, F.W., Eddington, A.S. and Davidson, C. (1920) A Determination of the Deflection of Light by the Sun's Gravitational Field, from Observations Made at the Total Eclipse of May 29, 1919. The Royal Society, 220, 291-333. https://doi.org/10.1098/rsta.1920.0009

[12] Pritchard, J. (n.d.) Gravitational Lensing. https://lweb.cfa.harvard.edu/ dfabricant/huchra/ay202/lectures/lecture12.pdf

[13] Bergmann, P.G. (1942) Introduction to the Theory of Relativity. Prentice-Hall, Inc., Hoboken. (Copyright 1942)

[14] Shapiro, I.I. (1964) Fourth Test of General Relativity. Physical Review Letters, 13, 789-791. https://doi.org/10.1103/PhysRevLett.13.789

[15] Will, C.M. (1993) Theory and Experiment in Gravitational Physics. Cambridge University Press, Cambridge.

[16] Shapiro, I.I., Ash, M.E., Ingalls, R.P., Smith, W.B., Campbell, D.B., Dyce, R.B., Jurgens, R.F. and Pettengill, G.H. (1971) Fourth Test of General Relativity: New Radar Result. Physical Review Letters, 26, 1132-1135.

https://doi.org/10.1103/PhysRevLett.26.1132

[17] Fomalont, E.B. and Kopeikin, S.M. (2003) The Measurement of the Light Deflection from Jupiter: Experimental Results. Astrophysical Journal, 598, 704-711. https://doi.org/10.1086/378785

[18] Fomalont, E.B., Kopeikin, S.M., Jones, D., Honma, M. and Titov, O. (2010) Recent VLBA/VERA/IVS Tests of General Relativity. Proceedings of the International Astronomical Union, 5, 291-295. arXiv: 0912.3421. https://doi.org/10.1017/S1743921309990536

[19] Bertotti, B., Iess, L. and Tortora, P. (2003) A Test of General Relativity Using Radio Links with the Cassini Spacecraft. Nature, 425, 374-376. https://doi.org/10.1038/nature01997

[20] Pound, R.V. and Snider, J.L. (1965) Effect of Gravity on Gamma Radiation. Physical Review, 140, Article No. B788. https://doi.org/10.1103/PhysRev.140.B788

[21] Gonzalez, H.J.I., Rebolo, R., Pasquini, L., Curto, G.L., Molaro, P., Caffau, E., et al. (2020) The Solar Gravitational Redshift from HARPS-LFC Moon Spectra-A Test of 
the General Theory of Relativity. Astronomy \& Astrophysics, 643, Article No. A146. https://doi.org/10.1051/0004-6361/202038937

[22] Wilhelm, K. and Dwivedi, B.N. (2014) On the Gravitational Redshift. New Astrono$m y, 31,8-13$. https://doi.org/10.1016/j.newast.2014.01.012 